\title{
The Outcomes of Trabectome Surgery in Patients with Low, Middle, and High Preoperative Intraocular Pressure
}

This article was published in the following Dove Press journal: Clinical Ophthalmology

\section{Naoki Tojo \\ Atsushi Hayashi}

Department of Ophthalmology, Graduate School of Medicine and Pharmaceutical Sciences, University of Toyama, Toyama, Japan
Correspondence: Atsushi Hayashi Department of Ophthalmology, Graduate School of Medicine and Pharmaceutical

Sciences, University of Toyama, 2630

Sugitani, Toyama 930-0194, Japan

Tel +8I-76-434-7363

Fax +8I-76-434-5037

Email ahayashi@med.u-toyama.ac.jp
Background: We compared the efficiency of trabectome surgery for patients with differing preoperative intraocular pressure (IOP) values in a single-facility retrospective study. We evaluated surgical outcomes based on three grades of preoperative IOP: high (hi-IOP, >26 $\mathrm{mmHg}$ ), middle (mid-IOP, 18-26 mmHg), and low (lo-IOP, $<18 \mathrm{mmHg}$ ).

Patients and Methods: We analyzed the cases of 204 eyes that underwent trabectome surgery and were followed $>2$ years. We defined failure as a reduction of IOP $<20 \%$ or requiring additional glaucoma surgery. We used 4 cutoff values $>21,>18,>15$, and $>12$ $\mathrm{mmHg}$. Other factors that may affect surgical outcomes were also investigated: age, central corneal thickness (CCT), history of selective laser trabeculotrabculoplasty (SLT), preoperative visual field, and simultaneous cataract surgery.

Results: Trabectome surgeries significantly decreased the IOP values from $23.0 \pm 7.2 \mathrm{mmHg}$ to $13.6 \pm 3.6 \mathrm{mmHg}$ at 2 years post-surgery. The mid-IOP group achieved significantly better surgical outcomes than the lo-IOP and hi-IOP groups with cutoff values 21,18 , and 15 $\mathrm{mmHg}$. A thin CCT and simultaneous cataract surgery were significantly related to better surgical outcomes with cutoff value 21.18 and $15 \mathrm{mmHg}$.

Conclusion: For the patients with preoperative IOP $<18 \mathrm{mmHg}$, it was difficult to decrease their IOP $>20 \%$ with trabectome surgery. Patients with preoperative IOP values $>26 \mathrm{mmHg}$ often required additional glaucoma surgery.

Keywords: trabectome, surgical outcome, intraocular pressure, factor, preoperative IOP

\section{Background}

The trabectome (Neomedix, Tustin, CA, US) was approved for clinical use by the US Food and Drug Administration in April 2004. ${ }^{1}$ An ab interno trabeculotomy with trabectome surgery is a minimally invasive glaucoma surgery (MIGS), as it spares the conjunctiva and requires only $1.7-\mathrm{mm}$ corneal incision. The trabectome ablates the trabecular meshwork and inner wall of the Schlemm's canal and provides the drainage of aqueous humor from the anterior chamber directly to a collector channel. It has been speculated that trabectome surgery can decrease the intraocular pressure (IOP) by decreasing the outflow resistance of the trabecular meshwork. $^{2}$

Many research groups have described the surgical outcomes of trabectome surgery. The reported mean values of 1-year postoperative IOP are $14.7-17.1 \mathrm{mmHg}^{3-7}$ It seems difficult to decrease the IOP to $<14 \mathrm{mmHg}$ with trabectome surgery. A preoperative IOP value $>21 \mathrm{mmHg}$ was considered to be appropriate for trabectome 
surgery, ${ }^{6,7}$ whereas another study indicated that eyes with low preoperative IOP values achieved better surgical outcomes than eyes with high preoperative IOP values. ${ }^{8}$ The question thus remains: which preoperative IOP values are actually appropriate for this surgery? We conducted the present study to address this issue. We divided eyes into three groups based on their preoperative IOP values and then compared the surgical outcomes after trabectome surgery.

\section{Patients and Methods}

\section{Patients}

This was a single-facility, nonrandomized, observational retrospective study. We analyzed the cases of 204 consecutive patients (246 eyes) who underwent a trabectome surgery at the University of Toyama and were followed $>2$ years. There were 42 patients who underwent trabectome in both eyes, and in these cases, we used only unilateral data of the eye that was operated earlier. All patients were recruited during the period from July 2014 to March 2018. All patients underwent a comprehensive ophthalmic examination including refraction, best corrected visual acuity, Goldmann gonioscopy, Goldmann applanation tonometry (GAT), fundus examination, optical coherence tomography (OCT) for the analysis of glaucoma, and automated perimetry (Humphrey Field Analyzer; Carl Zeiss Meditec, Dublin, CA) with the 30-2 Swedish Interactive Thresholding Algorithm standard. One glaucoma specialist (N.T.) diagnosed all of the cases of glaucoma and decided whether trabectome surgery should be performed. For patients who were still young and had a clear lens, we choose trabectome surgery alone. For elderly patients with cataracts, we choose trabectome and cataract surgery simultaneously. Because this was a retrospective study, we did not define clear surgical indications or clear criteria for simultaneous cataract surgery.

The research protocol was approved by the Institutional Review Board of the University of Toyama, and the procedures used conformed to the tenets of the Declaration of Helsinki. We explained the trabectome surgery and complications to all of the patients, and written informed consent for their data to be published was obtained from each patient. We did not include data for patients who underwent additional glaucoma surgery.

\section{Surgical Techniques}

All patients were operated on by one surgeon (N.T.) with the same surgical protocol, as described. ${ }^{8}$ A $1.7-\mathrm{mm}$ temporal clear corneal incision was created, and an ophthalmic viscosurgical device was injected into the anterior chamber for stability. Under the Swan Jacob gonioscopic lens view, the trabectome was inserted into Schlemm canal through the trabecular meshwork and ablating an approx. $120^{\circ}$ arc of the trabecular meshwork at only the nasal quadrant. For combination cases with cataract surgery, the cataract surgery was performed after the trabectome surgery. A 2.8-mm clear corneal incision was created at same incision for the trabectome, and we used Whitestar Signature ${ }^{\circledR}$ Pro Phaco system (Johnson \& Johnson, New Brunswick, NJ) for phacoemulsification. The wound was closed using a single $10-0$ nylon suture.

\section{Post-Operative Medication}

The postoperative medications used were topical antibiotics, steroids, non-steroidal anti-inflammatory drugs, and $2 \%$ pilocarpine for all patients. Topical antibiotics, steroids and pilocarpine were used for 1 month. Topical nonsteroidal anti-inflammatory drugs were used for 3 months. The purpose of the pilocarpine was to avoid peripheral anterior synechia (PAS). All patients continued to use their preoperative glaucoma medications after the trabectome surgery. Continued treatment of postoperative glaucoma medications is the recommended in Japan. We counted a fixed-combination agent as two medications.

\section{Definition of Success}

We defined the patients" "preoperative IOP" as the mean of the patient's IOP values measured with GAT at two visits just before the trabectome surgery. We divided the patients into three groups based on their preoperative IOP values as follows: $<18 \mathrm{mmHg}$ as the lo-IOP group, 18-26 $\mathrm{mmHg}$ as the mid-IOP group, and $>26 \mathrm{mmHg}$ as the hiIOP group. Failure was defined a priori as IOP of more than cutoff value or less than $20 \%$ reduction below baseline on 2 consecutive follow-up visits after 3 months. Cases that required additional glaucoma surgery, developed phthisis, or developed loss of light perception were also considered failures. All patients received postoperative tolerance glaucoma medications. The patients with deterioration of visual field due to insufficient IOP decrease and with non-IOP lowering effects after surgery were undergone additional glaucoma surgery. The decision on additional glaucoma surgery was dependent on one glaucoma specialist (N.T). 
We used the cutoff IOP values for surgical success as $\leqq 21($ Criteria A), $\leqq 18$ (Criteria B), $\leqq 15$ (Criteria C), and $\leqq 12$ (Criteria D) $\mathrm{mmHg}$.

\section{Other Factors That Affect Surgical Outcomes}

The main outcome of this study was the success rate of each of the three preoperative IOP groups as Kaplan-Meier analysis. We also investigated the following factors that can affect surgical outcomes: patient age (young $<70$ years old, elderly $\geqq 70$ years old) central corneal thickness (CCT) (thin $<525 \mu \mathrm{m}$, thick $\geqq 525 \mu \mathrm{m}$ ), mean deviation (MD) (mild, $\geqq-14 \mathrm{~dB}$, severe $<-14 \mathrm{~dB}$ ) (in the results of the Humphrey visual field just before the trabectome surgery), history of selective laser trabeculoplasty (SLT), and type of surgical method (trabectome alone (Single surgery) or combined simultaneous cataract surgery (Triple surgery)). We classified the types of glaucoma into the following four groups: primary open angle glaucoma (POAG), primary angle closure glaucoma (PACG), pseudo-exfoliation glaucoma (PEXG), and other secondary glaucoma (SG).

\section{Statistical Analysis}

We used JMP Pro 14software (SAS, Cary, NC) to analyze our findings. A paired $t$-test and Fisher's exact test were used for the comparisons of the three patient groups. We used the Wilcoxon signed-rank test to compare the same patients' data and the Kaplan-Meier method to compare the groups' success rates along with the log-rank test. The factors that may affect surgical outcomes were examined by a multivariate analysis. Statistical significance was defined at $\mathrm{p}<0.05$.

\section{Results}

\section{The Ophthalmic Data and Postoperative IOP Values of All Patients}

After the inclusion criteria were applied, a final total of 204 eyes were analyzed. All surgeries were performed successfully. Thirty-nine patients underwent surgery for both eyes. Table 1 summarizes the patients' baseline characteristics, classified into the three IOP groups. The mean follow-up period was $39.2 \pm 12.1$ months. The lo-IOP group ( $\mathrm{n}=55 \sim 27 \%$ ) included many elderly patients, many POAG patients, no SG patient, worse MD values in the visual field test, and many patients with simultaneous cataract (Triple) surgery. The mid-IOP group $(n=91$, $\sim 45 \%)$ had better MD values. The hi-IOP group $(\mathrm{n}=58$, $\sim 28 \%$ ) had many younger patients, few POAG patients, many SG patients, and few patients with simultaneous cataract surgery.

\section{Postoperative IOP Values}

The IOP groups' mean postoperative IOP values are provided in Table 2. The patients' preoperative glaucoma medications were continued after their surgery. The results of our analyses revealed that trabectome surgery significantly reduced the patients' IOP values at all time points. In addition, the mean postoperative IOP value in the loIOP group was significantly lower than that other group at 3 years $(\mathrm{p}=0.0057)$. The mean postoperative IOP values of the mid-IOP and hi-IOP groups were not significantly different at any time point.

The IOP reduction values are shown in Table 3 . Among the patients without additional glaucoma surgery, the hi-IOP group exhibited the highest reduction in IOP after trabectome surgery.

\section{Surgical Outcomes}

Figure 1 illustrates the results of the Kaplan-Meier analysis of surgical outcomes with cutoff IOP value $(21,18,15$, and $12 \mathrm{mmHg}$ ).

Figure 1A shows the results with Criteria A (cutoff value $\leqq 21 \mathrm{mmHg}$ ). In the lo-IOP group, the success rates at 1, 2, and 3 years were $69.1 \%, 50.9 \%$, and $46.7 \%$, respectively. In the mid-IOP group, the corresponding values were $86.8 \%$, $78.9 \%$, and $78.9 \%$, and those of the hi-IOP group were $55.2 \%, 48.0 \%$, and $45.2 \%$, respectively.

Figure 1B shows the results with Criteria B (cutoff value $\leqq 18 \mathrm{mmHg}$ ). In the lo-IOP group, the success rates at 1,2 , and 3 years were $69.1 \%, 50.9 \%$, and $46.7 \%$, respectively. In the mid-IOP group, the corresponding values were $86.8 \%$, $78.9 \%$, and $74.4 \%$, and those of the hi-IOP group were $51.7 \%, 46.4 \%$, and $40.6 \%$, respectively.

Figure $1 \mathrm{C}$ shows the results with Criteria $\mathrm{C}$ (cutoff value $\leqq 15 \mathrm{mmHg}$ ). In the lo-IOP group, the success rates at 1,2 , and 3 years were $69.1 \%, 49.1 \%$, and $44.8 \%$, respectively. In the mid-IOP group, the corresponding values were $80.2 \%$, $65.5 \%$, and $61.6 \%$, and those of the hi-IOP group were $55.0 \%, 46.6 \%$, and $34.3 \%$, respectively.

Figure 1D shows the results with Criteria D (cutoff value $\leqq 12 \mathrm{mmHg}$ ). In the lo-IOP group, the success rates at 1,2 , and 3 years were $54.6 \%, 38.2 \%$, and $33.4 \%$, respectively. In the mid-IOP group, the corresponding values were $50.6 \%$, 
Table I Ophthalmic Data of All 204 Eyes

\begin{tabular}{|c|c|c|c|c|c|}
\hline & All (204 Eyes) & Lo-IOP (55 Eyes) & Mid-IOP (9 I Eyes) & Hi-IOP (58 Eyes) & p-value \\
\hline Age, yrs & $70.1 \pm 11.1$ & $70.9 \pm 8.4$ & $71.9 \pm 9.2$ & $66.6 \pm 14.7$ & 0.015 \\
\hline Male/female & $116 / 88$ & $22 / 23$ & $50 / 41$ & $33 / 25$ & 0.836 \\
\hline Follow-up period, mos. & $39.2 \pm 12.1$ & $40.2 \pm 10.5$ & $38.6 \pm 12.8$ & $39.1 \pm 12.3$ & 0.749 \\
\hline$M D, d B$ & $-11.7 \pm 10.3$ & $-11.9 \pm 6.3$ & $-9.7 \pm 6.7$ & $-9.7 \pm 6.7$ & 0.143 \\
\hline \multicolumn{6}{|l|}{ Type of glaucoma: } \\
\hline POAG, $n$ & 62 & 31 & 30 & 9 & 0.0056 \\
\hline PACG, $n$ & 4 & I & 2 & I & 0.978 \\
\hline PEXG, $n$ & 113 & 23 & 53 & 29 & 0.607 \\
\hline SG, $n$ & 25 & 0 & 6 & 19 & $<0.0001$ \\
\hline History of SLT, A/NA & $19 / 185$ & $4 / 51$ & $7 / 84$ & $8 / 50$ & 0.405 \\
\hline Single/triple surgery & $99 / 105$ & $21 / 34$ & $38 / 53$ & $40 / 18$ & 0.0009 \\
\hline $\mathrm{CCT}, \mu \mathrm{m}$ & $527 \pm 35$ & $524 \pm 33$ & $525 \pm 38$ & $533 \pm 34$ & 0.306 \\
\hline Glaucoma medications & $3.5 \pm 1.0$ & $3.6 \pm 1.2$ & $3.5 \pm 1.0$ & $3.6 \pm 1.0$ & 0.862 \\
\hline Preoperative IOP, mmHg & $23.0 \pm 7.2$ & $15.3 \pm 2.7$ & $22.0 \pm 2.0$ & $31.9 \pm 5.5$ & $<0.0001$ \\
\hline
\end{tabular}

Abbreviations: MD, mean deviation; mos, months; PACG, primary angle closure glaucoma; PEXG, pseudo-exfoliation glaucoma; POAG, primary open angle glaucoma.

Table 2 Pre- and Post-Operative IOP Values

\begin{tabular}{|l|l|l|l|l|}
\hline IOP, $\mathbf{m m H g}$ & All (n) & Lo-IOP (n) & Mid-IOP (n) & Hi-IOP (n) \\
\hline Pre-op. IOP & $23.0 \pm 7.2(204)$ & $15.3 \pm 2.7(55)$ & $22.0 \pm 2.0(9 I)$ & $31.9 \pm 5.5(58)$ \\
\hline $\begin{array}{l}\text { Post-op. IOP } \\
\text { I yr }\end{array}$ & $13.0 \pm 3.4(166)$ & $12.5 \pm 3.7(52)$ & & \\
2 yrs & $13.6 \pm 3.6(154)$ & $12.7 \pm 3.7(46)$ & $13.0 \pm 2.9(80)$ & $13.5 \pm 4.0(34)$ \\
3 yrs & $12.5 \pm 3.2(97)$ & $11.2 \pm 2.7(36)$ & $13.4 \pm 3.2(46)$ & $13.6 \pm 3.1(30)$ \\
4 yrs & $12.7 \pm 3.4(45)$ & $11.3 \pm 2.1(16)$ & $13.8 \pm 3.8(24)$ & $13.1 \pm 3.4(15)$ \\
5 yrs & $13.2 \pm 4.0(20)$ & $10.5 \pm 1.9(4)$ & $14.0 \pm 4.4(14)$ & $12.2 \pm 2.8(5)$ \\
\hline
\end{tabular}

Abbreviations: IOP, intraocular pressure, Pre-op., pre-operative; Post-op., post-operative; SG, other secondary glaucoma; Single, trabectome surgery alone; SLT, selective laser trabeculoplasty; Triple, trabectome surgery with simultaneous cataract surgery.

Table 3 The Reduction in IOP Values Compared with the Preoperative IOP

\begin{tabular}{|l|l|l|l|}
\hline Reduction Rate (\%) & Lo-IOP (n) & Mid-IOP (n) & Hi-IOP (n) \\
\hline 1 yr & $16.2 \pm 24.2(52)$ & $42.6 \pm 14.2(80)$ & $56.1 \pm 14.4(34)$ \\
2 yrs & $15.1 \pm 22.2(46)$ & $37.1 \pm 16.9(78)$ & $54.8 \pm 14.8(30)$ \\
3 yrs & $23.4 \pm 19.9(36)$ & $39.1 \pm 15.4(46)$ & $57.8 \pm 13.6(15)$ \\
4 yrs & $15.6 \pm 25.1(16)$ & $38.3 \pm 17.3(24)$ & $62.4 \pm 11.3(5)$ \\
5 yrs & $39.8 \pm 8.8(4)$ & $38.8 \pm 20.3(14)$ & $67.1 \pm 5.7(2)$ \\
\hline
\end{tabular}

$38.3 \%$, and $33.1 \%$, and those of the hi-IOP group were $34.5 \%, 22.4 \%$, and $13.8 \%$, respectively.

With Criteria A, B, and C, mid-IOP group was significantly better surgical outcomes than other groups. With Criteria D, hi-IOP group was significantly worse than other groups, the surgical outcomes of lo-IOP and midIOP group were about the same results.

The hi-IOP group thus achieved worse surgical outcomes within the first 12 months after surgery. In hi-IOP group, there were 30 eyes required additional surgeries, 25 eyes of them $(83.3 \%)$ underwent additional glaucoma surgeries within 12 months. Over the long term, lo-IOP group's surgical outcomes became worse than those of the hi-IOP group with Criteria A and B.

The reasons for failure of the surgery are depicted in Figure 2. White means success. Black means additional glaucoma surgery. Gray means IOP reduction $<20 \%$ or more than cut off IOP value. In the lo-IOP group, there 

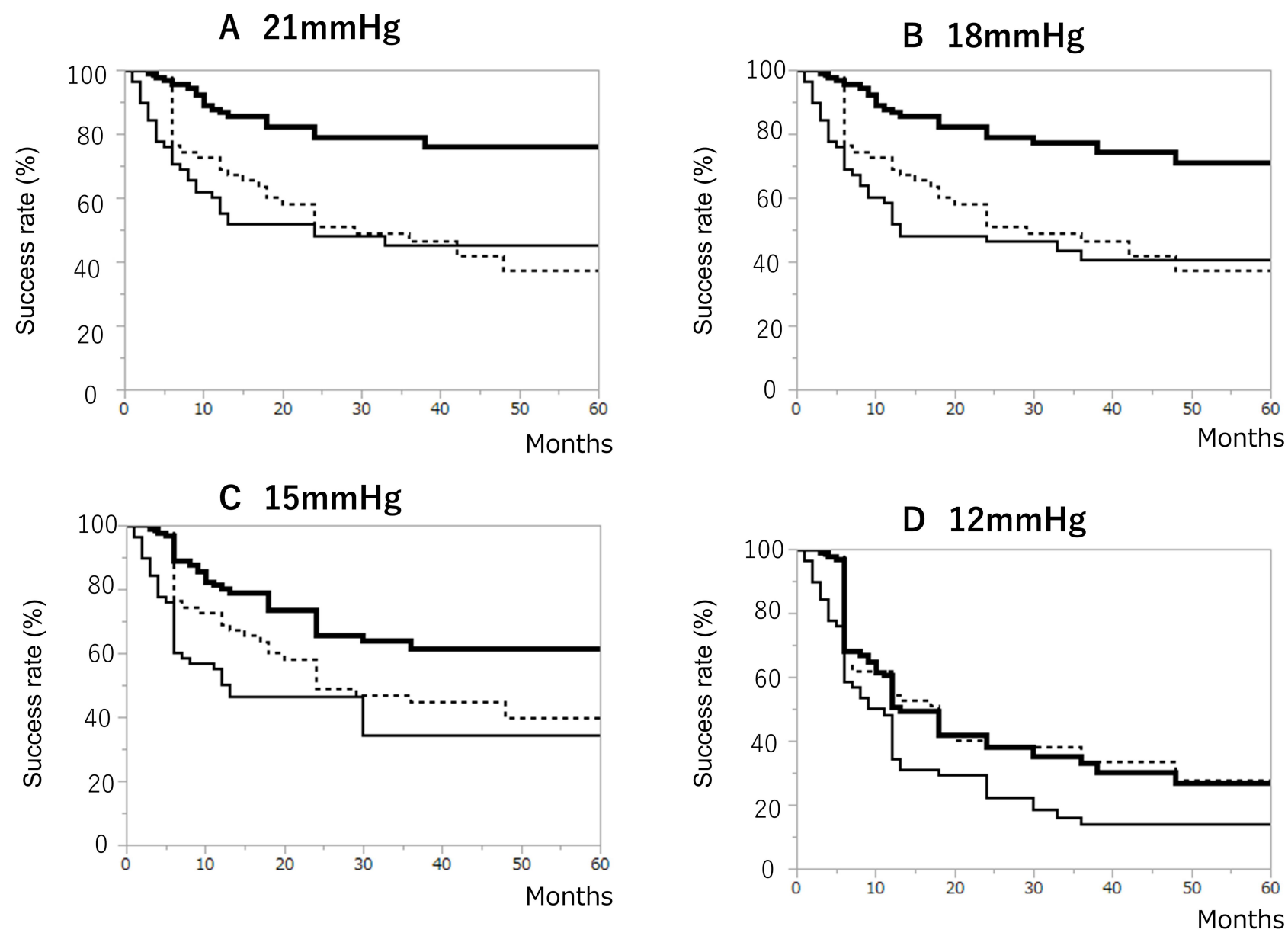

Figure I The Kaplan-Meier analysis of surgical outcomes. Dotted line: The lo-IOP group's results. Bold line: The mid-IOP results. Normal line: The hi-IOP results. (A) The Kaplan-Meier analysis of surgical outcomes with Criteria A. The lo-IOP versus the mid-IOP $(p<0.00 I)$. The mid-IOP versus the hi-IOP $(\mathrm{p}<0.00 \mathrm{I})$. The lo-IOP versus the hiIOP $(p=0.569)$. (B) The Kaplan-Meier analysis of surgical outcomes with Criteria $B$. The lo-IOP versus the mid-IOP $(p<0.00 I)$. The mid-IOP versus the hi-IOP $(p<0.00 I)$. The Io-IOP versus the hi-IOP $(\mathrm{P}=0.329)$. (C) The Kaplan-Meier analysis of surgical outcomes with Criteria $C$. The lo-IOP versus the mid-IOP $(\mathrm{P}=0.0263)$. The mid-IOP versus the hi-IOP $(p=0.0002)$. The lo-IOP versus the hi-IOP $(p=0.135)$. (D) The Kaplan-Meier analysis of surgical outcomes with Criteria $D$. The lo-IOP versus the mid-IOP $(p=0.885)$. The mid-IOP versus the hi-IOP $(p=0.0047)$. The lo-IOP versus the hi-IOP $(p=0.0083)$.

were $32.7-43.6 \%$ cases that the reduction rate $<20 \%$ or increasing more than the cutoff value. In the mid-IOP group, the success rate was the highest among the three IOP groups. In the hi-IOP group, cases of $52.7 \%$ required additional glaucoma surgery.

\section{Analysis of Factors Related to Surgical Outcomes}

The results of the multivariate analysis for the surgical success factors are shown in Table 4 (Criteria A), Table 5 (Criteria B), Table 6 (Criteria C), and Table 7 (Criteria D). Thin CCT (525 $\mu \mathrm{m}$ or less) was significant factors for better surgical outcomes at any cutoff IOP value. With Criteria A, B, and C, simultaneous cataract surgery was significant factors for better surgical outcomes. With Criteria A, B, and C, the surgical outcomes of mid-IOP group were significantly better.

\section{Postoperative Complications}

An anterior chamber hemorrhage is the most common complication of trabectome surgery. All patients were observed intraoperative hyphema that is due to reverse flow from episcleral venous. There was no patient who needed irrigation of the anterior chamber additionally. No serious vision-threatening complications (eg, hypotonic maculopathy, rhegmatogenous retinal detachment, or endophthalmitis) occurred.

\section{Discussion}

The results of our present retrospective analyses of 204 eyes demonstrated that trabectome surgery significantly reduced the patients' IOP, and the patients with preoperative IOP values in the mid-range (ie, $18-26 \mathrm{mmHg}$ ) achieved a better success rate than the patients with low $(<18 \mathrm{mmHg})$ or high $(>26 \mathrm{mmHg})$ preoperative IOP values. The mean postoperative IOP value in 


\section{A $21 \mathrm{mmHg}$}

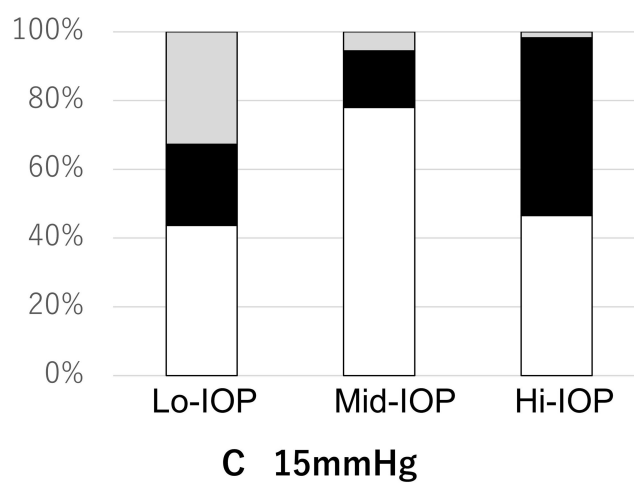

\section{B $18 \mathrm{mmHg}$}

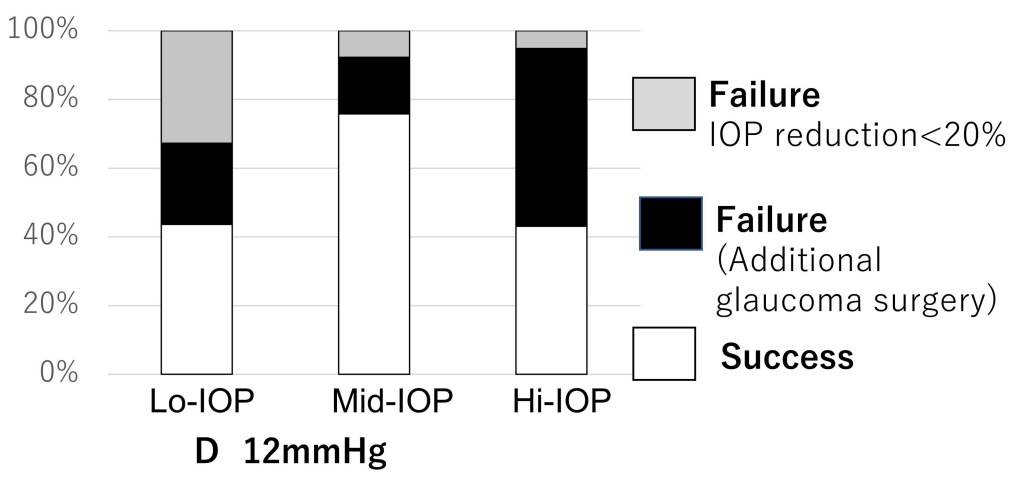

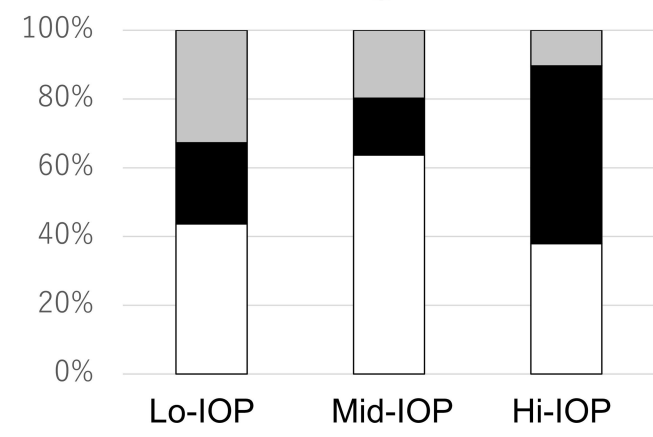

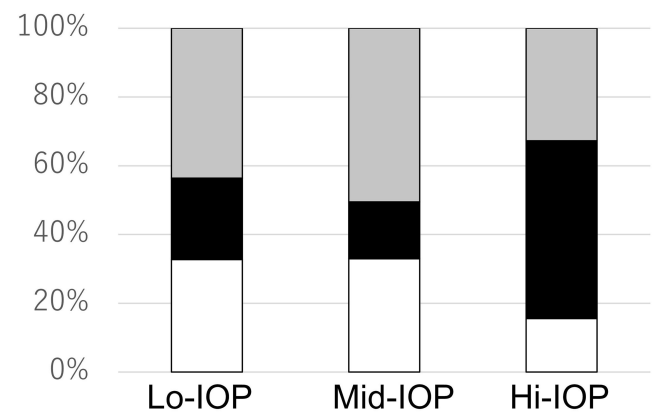

Figure 2 The reasons for failure. White: Success. Black: Additional glaucoma surgery. Gray: IOP reduction $<20 \%$ or more than cut off IOP value. (A-D) show the results of Criteria A, B, C and D, respectively.

the lo-IOP group was significantly lower than those of the other two groups. The factors that significantly affected the surgical outcomes in this patient series were the thickness of CCT and simultaneous cataract surgery.

It has been reported that thin CCT, simultaneous cataract surgery, no history of SLT, elderly status, good visual field results, and pseudo-exfoliation glaucoma are factors for better outcomes. ${ }^{7-12}$ In the present study, the patients' preoperative IOP was not a factor influencing the surgical outcomes. Simultaneous cataract surgery was reported to be associated with better surgical outcomes in several studies. $^{6-9}$ Simultaneous cataract surgery can widen the anterior chamber angle, thus providing better surgical outcomes. Esfandiari et al reported that a thin CCT was associated with better surgical outcomes. ${ }^{10}$ Our present findings agree with this report. The reason why a thin CCT results in better surgical outcomes is not yet known.

It should be noted that surgical outcomes can change with different definitions of success. For example, if the success of trabectome surgery is defined as a $>30 \%$ reduction in IOP compared to preoperative IOP, our present lo-IOP group would have the worst surgical outcomes. One of the most important clinical findings is a mean postoperative IOP value at $12-14 \mathrm{mmHg}$, whether the patient's preoperative IOP was

Table 4 The Results of the Multivariate Analysis for Surgical Success Factors $21 \mathrm{mmHg}$

\begin{tabular}{|l|l|l|l|l|}
\hline & OR & $\mathbf{9 5 \%}$ Lower Cl & $\mathbf{9 5 \%}$ Upper Cl & p-value \\
\hline Age (young/old) & 1.083 & 0.450 & 2.454 & 0.848 \\
MD (severe/mild) & 1.204 & 0.567 & 2.654 & 0.645 \\
CCT (Thick/thin) & 2.657 & 1.245 & 5.668 & 0.0115 \\
Preoperative IOP (Hi/Mid) & 4.690 & 1.825 & 12.052 & 0.0013 \\
Preoperative IOP (Hi/Lo) & 1.398 & 0.509 & 3.834 & 0.517 \\
Preoperative IOP (Lo/Mid) & 6.555 & 2.705 & 15.885 & $<0.0001$ \\
Gender (male/female) & 1.768 & 0.826 & 3.783 & 0.142 \\
Surgical method (Single/Triple) & 2.566 & 1.176 & 5.602 & 0.018 \\
History of SLT (A/NA) & 0.785 & 0.203 & 3.026 & 0.725 \\
\hline
\end{tabular}

Abbreviations: $\mathrm{CCT}$, central corneal thickness; $\mathrm{Cl}$, confidence interval; MD, mean deviation; OR, odds ratio; SLT, selective laser trabeculoplasty. 
Table 5 The Results of the Multivariate Analysis for Surgical Success Factors $18 \mathrm{mmHg}$

\begin{tabular}{|l|l|l|l|l|}
\hline & OR & $\mathbf{9 5 \%}$ Lower Cl & $\mathbf{9 5 \%}$ Upper Cl & $\mathbf{p - v a l u e ~}$ \\
\hline Age (young/old) & 1.025 & 0.455 & 2.308 & 0.952 \\
MD (severe/mild) & 0.999 & 0.456 & 2.184 & 0.996 \\
CCT (Thick/thin) & 2.500 & 1.193 & 5.242 & 0.0152 \\
Preoperative IOP (Hi/Mid) & 4.756 & 1.899 & 11.912 & 0.009 \\
Preoperative IOP (Hi/Lo) & 1.159 & 0.427 & 3.143 & 0.772 \\
Preoperative IOP (Lo/Mid) & 5.512 & 2.319 & 13.099 & 0.001 \\
Gender (male/female) & 1.952 & 0.921 & 4.138 & 0.0809 \\
Surgical method (Single/Triple) & 2.490 & 1.156 & 5.361 & 0.0198 \\
History of SLT (A/NA) & 1.697 & 0.433 & 6.645 & 0.448 \\
\hline
\end{tabular}

Abbreviations: CCT, central corneal thickness; Cl, confidence interval; MD, mean deviation; OR, odds ratio; SLT, selective laser trabeculoplasty.

Table 6 The Results of the Multivariate Analysis for Surgical Success Factors $15 \mathrm{mmHg}$

\begin{tabular}{|l|l|l|l|l|}
\hline & OR & 95\% Lower Cl & 95\% Upper CI & p-value \\
\hline Age (young/old) & 1.356 & 0.623 & 2.955 & 0.443 \\
MD (severe/mild) & 1.288 & 0.583 & 2.588 & 0.589 \\
CCT (Thick/thin) & 2.124 & 1.046 & 4.313 & 0.0371 \\
Preoperative IOP (Hi/Mid) & 2.813 & 1.163 & 6.808 & 0.0218 \\
Preoperative IOP (Hi/Lo) & 0.877 & 0.323 & 2.385 & 0.798 \\
Preoperative IOP (Lo/Mid) & 2.488 & 1.105 & 5.516 & 0.0276 \\
Gender (male/female) & 1.759 & 0.918 & 3.766 & 0.105 \\
Surgical method (Single/Triple) & 2.467 & 1.193 & 5.102 & 0.0148 \\
History of SLT (A/NA) & 2.388 & 0.596 & 9.565 & 0.219 \\
\hline
\end{tabular}

Abbreviations: CCT, central corneal thickness; $\mathrm{Cl}$, confidence interval; MD, mean deviation; OR, odds ratio; SLT, selective laser trabeculoplasty.

Table 7 The Results of the Multivariate Analysis for Surgical Success Factors $12 \mathrm{mmHg}$

\begin{tabular}{|l|l|l|l|l|}
\hline & OR & $\mathbf{9 5 \%}$ Lower Cl & $\mathbf{9 5 \%}$ Upper Cl & $\mathbf{p - v a l u e ~}$ \\
\hline Age (young/old) & 2.104 & 0.851 & 5.200 & 0.107 \\
MD (severe/mild) & 0.909 & 0.391 & 2.114 & 0.824 \\
CCT (Thick/thin) & 3.928 & 1.707 & 9.036 & 0.0013 \\
Preoperative IOP (Hi/Mid) & 1.544 & 0.524 & 4.546 & 0.431 \\
Preoperative IOP (Hi/Lo) & 0.469 & 0.145 & 1.516 & 0.206 \\
Preoperative IOP (Lo/Mid) & 0.724 & 0.313 & 1.678 & 0.452 \\
Gender (male/female) & 0.754 & 0.344 & 1.654 & 0.481 \\
Surgical method (Single/Triple) & 1.963 & 0.877 & 4.394 & 0.0101 \\
History of SLT (A/NA) & 1.606 & 0.305 & 8.670 & 0.576 \\
\hline
\end{tabular}

Abbreviations: $\mathrm{CCT}$, central corneal thickness; $\mathrm{Cl}$, confidence interval; MD, mean deviation; OR, odds ratio; SLT, selective laser trabeculoplasty.

low or high. The episcleral venous pressure was reported to be 8.6-9.4 mmHg, ${ }^{13,14}$ considering that the postoperative IOP can be higher than the episcleral venous pressure, it would be difficult to reduce the IOP to $<10 \mathrm{mmHg}$ with surgery to remove the resistance due to the trabecular meshwork outflow. ${ }^{15}$

The postoperative IOP values obtained in our present investigation are $1-2 \mathrm{mmHg}$ lower than those in previous reports. The reason for this is that our patients were using a greater number of glaucoma medications compared to the past patient series. Our patients also continued to use their preoperative glaucoma medications after undergoing the trabectome or combination surgery. The mean number of glaucoma medications was thus not decreased, but it is considered equivalent to the previous results regarding glaucoma medications. In our lo-IOP group, the mean 
postoperative IOP values were significantly lower than those of the other two groups, and this might be because the native episcleral venous pressure was low.

In the lo-IOP group, trabectome surgery lowered the patients' IOP by approx. $2.5 \mathrm{mmHg}$, and the IOP reduction rate was $14.2 \%$ at 2 years postsurgery. The Collaborative Normal-Tension Glaucoma Study Group reported that normal-tension glaucoma requires an IOP reduction rate of $\geq 30 \% .{ }^{16}$ In the present study, trabectome surgery alone for the lo-IOP group might not be sufficiently effective. There were 37 eyes with unsuccessful results based on a $<20 \%$ reduction of IOP, and 12 of these 37 eyes needed additional glaucoma surgery. It might be advisable to choose filtration surgery for cases with low preoperative IOP. Considering our patients' mean postoperative IOP values and the standard deviation, a good indication for trabectome surgery is a preoperative IOP value $\geq 16 \mathrm{mmHg}$.

In the mid-IOP group, the mean IOP reduction was $>30 \%$. There were 39 eyes with unsuccessful outcomes and 33 eyes that needed additional glaucoma surgery. The surgical results of this group were the best, as the mean IOP reduction at $>30 \%$ was due to the patients' higher preoperative IOP values compared to those of the lo-IOP group, and the percentage of eyes that required additional glaucoma surgery was less than that of the hi-IOP group.

In the hi-IOP group, the mean IOP reduction was $>50 \%$. The trabectome surgery lowered the IOP values to approx. $20 \mathrm{mmHg}$. In general, the higher the preoperative IOP is, the higher the IOP reduction will be. Focusing only on the reduction of IOP, a trabectome surgery is indicated for patients with high preoperative IOP values, but the rate of additional glaucoma surgery in the present hi-IOP group was high ( $\mathrm{n}=30$ eyes). The reasons for this result are that the hi-IOP group had many patients with a thick CCT and patients who underwent trabectome surgery alone; trabectome surgery alone and thick CCT are factors that often result in surgical failure. We did not use corrected IOP with CCT.

Esfandiari et al reported that high preoperative IOP was one of the factors for worse surgical outcomes. ${ }^{10}$ In the present analyses, additional glaucoma surgery had been performed early after surgery; 27 eyes (90\%) required the additional surgery within 1 year. In shortterm surgical outcomes, high preoperative IOP values can easily lead to failure. The present patients whose IOP was sufficiently reduced tended to maintain good results over a long period of time. High preoperative IOP values might not be a good indication for trabectome surgery. However, regarding MIGS, trabectome surgery could be one of the options to consider before filtration surgery. It was reported that MIGS that can be performed with conjunctival preservation did not affect the surgical results of filtration surgery. ${ }^{17}$

Our study has some limitations. We divided into three groups as high, middle, and low; however, there was no evidence of the cutoff value. Despite surgical outcomes could vary depending on type of glaucoma, we included various types of glaucoma. We included patients who underwent trabectome surgery alone and patients who underwent trabectome and cataract surgery. The effect of lowering IOP due to cataract surgery cannot be ignored. It was retrospective, and the recruited patients of the three IOP groups showed significant differences in age, MD value, type of glaucoma, and type of surgical method. The reason why the lo-IOP group contained many elderly patients and advanced visual field values might be because the surgeons were hesitant to perform surgery due to the low IOP. Our hi-IOP group contained many SG patients, perhaps because SG often causes high IOP. We should compare patients with the same glaucoma type, or who have undergone the same surgical methods. In addition, there is no clear definition of surgical indications and indications for additional glaucoma surgery. The IOP measurement time points are not defined. (We did not take into account the IOP fluctuation.) The mean follow-up period in this study was 39 months, and longer follow-ups are desired. During the multivariate analysis, we divided the influencing factors into the two groups, but there was no evidence of the cutoff values.

This study had high chances of having regression to the mean. Even with no intervention, eyes with pre-high and low IOP values will have lower and higher readings, respectively. We analyzed using various cutoff values. Although there are many reports of surgical outcome of trabectome, some are including many patients with high preoperative IOP, and some are including many patients with low preoperative IOP. So it is difficult to compare such reports. We expect that our study will be useful for comparing such reports.

The mean postoperative IOP values of our patient tended to settle at approx. $12-14 \mathrm{mmHg}$, regardless of the preoperative IOP value with glaucoma medications. The mid-IOP range (18-26 $\mathrm{mmHg}$ ) could achieve a better benefit from trabectome surgery. It is also expected that a thin CCT and simultaneous cataract surgery could result in better surgical results. 


\section{Code Availability}

We used JMP Version 11 for statistical analysis.

\section{Data Sharing Statement}

The datasets during and/or analysed during the current study available from the corresponding author on reasonable request.

\section{Ethics Approval}

All procedures performed in this study involving human participants were in accordance with the ethical standards of the institutional and/or national research committee and with the 1964 Helsinki Declaration and its later amendments or comparable ethical standards.

\section{Consent to Participate}

Each patient in this study provided informed consent after a thorough explanation of the study.

\section{Consent for Publication}

We obtained consent for publication from each patient.

\section{Author Contributions}

All authors contributed to data analysis, drafting or revising the article, have agreed on the journal to which the article will be submitted, gave final approval of the version to be published, and agree to be accountable for all aspects of the work.

\section{Funding}

No funding was received for this research.

\section{Disclosure}

The authors declare that they have no affiliations with or involvement in any organization or entity with any financial interest (such as honoraria; educational grants; participation in speakers' bureaus; membership, employment, consultancies, stock ownership, or other equity interest; and expert testimony or patent-licensing arrangements), or non-financial interest (such as personal or professional relationships, affiliations, knowledge or beliefs) in the subject matter or materials discussed in this manuscript. The authors report no conflicts of interest in this work.

\section{References}

1. Minckler DS, Baerveldt G, Ramirez Alfaro M, Francis BA. Clinical results with the trabectome for treatment of open-angle glaucoma. Ophthalmology. 2005;112(6):1540. doi:10.1016/j.ophtha.2004.12.043

2. Francis BA, Winarko J. Ab interno schlemm's canal surgery: trabectome and iStent. Dev Ophthalmol. 2012;50:125-136. doi:10.1159/ 000334794

3. Akil H, Chopra V, Huang A, Loewen N, Noguchi J, Francis BA. Clinical results of ab interno trabeculotomy using the trabectome in patients with pigmentary glaucoma compared to primary open angle glaucoma. Clin Exp Ophthalmol. 2016;44(7):563-569. doi:10.1111/ceo.12737

4. Bussel II, Kaplowitz K, Schuman JS, Loewen NA. Trabectome study group. Outcomes of ab interno trabeculectomy with the trabectome by degree of angle opening. Br J Ophthalmol. 2015;99(7):914-919. doi:10.1136/bjophthalmol-2014-305577

5. Ting JLM, Damji KF, Stiles MC. Trabectome Study Group. Ab interno trabeculectomy: outcomes in exfoliation versus primary open-angle glaucoma. $J$ Cataract Refract Surg. 2012;38 (2):315-323. doi:10.1016/j.jcrs.2011.08.043

6. Ahuja Y, Ma Khin Pyi S, Malihi M, Hodge DO, Sit AJ. Clinical results of $a b$ interno trabeculotomy using the trabectome for open-angle glaucoma: the mayo clinic series in Rochester, Minnesota. Am J Ophthalmol. 2013;156(5):927-935. doi:10.1016/j. ajo.2013.06.001

7. Shoji N, Kasahara M, Iijima A, et al. Short-term evaluation of trabectome surgery performed on Japanese patients with open-angle glaucoma. Jpn J Ophthalmol. 2016;60(3):156-165. doi:10.1007/ s10384-016-0433-5

8. Tojo N, Abe S, Hayashi A. Factors that influence of trabectome surgery for glaucoma patients. J Glaucoma. 2017;26(9):835-844. doi:10.1097/IJG.0000000000000743

9. Okeke CO, Miller-Ellis E, Rojas M. The trabectome study group. Trabectome success factors. Medicine. 2017;96(24):e7061. doi:10.1097/MD.0000000000007061

10. Esfandiari H, Shah P, Torkian P, et al. Five-year clinical outcomes of combined phacoemulsification and trabectome surgery at a single glaucoma center. Graefes Arch Clin Exp Ophthalmol. 2019;257 (2):357-362. doi:10.1007/s00417-018-4146-y

11. Loewen RT, Roy P, Parikh HA, Dang Y, Schuman JS, Loewen NA. Impact of a glaucoma severity index on results of trabectome surgery: larger pressure reduction in more severe glaucoma. PLoS One. 2016;11(3):e0151926. doi:10.1371/journal.pone.0151926

12. Avar M, Jordan JF, Neuburger M, et al. Long-term follow-up of intraocular pressure and pressure-lowering medication in patients after ab-interno trabeculectomy with the trabectome. Graefes Arch Clin Exp Ophthalmol. 2019;257(5):997-1003. doi:10.1007/s00417019-04259-5

13. Sultan M, Blondeau P. Episcleral venous pressure in younger and older subjects in the sitting and supine positions. J Glaucoma. 2003;12(4):370-373. doi:10.1097/00061198-200308000-00013

14. Toris CB, Yablonski ME, Wang Y-L, Camras CB. Aqueous humor dynamics in the aging human eye. Am J Ophthalmol. 1999;127 (4):407-412. doi:10.1016/S0002-9394(98)00436-X

15. Sit AJ, McLaren JW. Measurement of episcleral venous pressure. Exp Eye Res. 2011;93(3):291-298. doi:10.1016/j.exer.2011.05.003

16. Collaborative Normal-Tension Glaucoma Study Group. Comparison of glaucomatous progression between untreated patients with normal-tension glaucoma and patients with therapeutically reduced intraocular pressures. Am J Ophthalmol. 1998;126(4):487-497. doi:10.1016/s0002-9394(98)00223-2.

17. Jea SY, Mosaed S, Vold SD, Rhee DJ. Effect of a failed Trabectome on subsequent trabeculectomy. J Glaucoma. 2012;21(2):71-75. doi:10.1097/IJG.0b013e31820bcfda 


\section{Publish your work in this journal}

Clinical Ophthalmology is an international, peer-reviewed journal covering all subspecialties within ophthalmology. Key topics include: Optometry; Visual science; Pharmacology and drug therapy in eye diseases; Basic Sciences; Primary and Secondary eye care; Patient Safety and Quality of Care Improvements. This journal is indexed on PubMed

Submit your manuscript here: https://www.dovepress.com/clinical-ophthalmology-journal
Central and CAS, and is the official journal of The Society of Clinical Ophthalmology (SCO). The manuscript management system is completely online and includes a very quick and fair peer-review system, which is all easy to use. Visit http://www.dovepress.com/ testimonials.php to read real quotes from published authors. 